\title{
Diagnostic ability of Japan Narrow-Band Imaging Expert Team classification for colorectal lesions by magnifying endoscopy with blue laser imaging versus narrow-band imaging
}

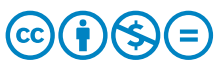

\begin{abstract}
Authors Keisuke Hori' ${ }^{1}$, Tomonori Yano ${ }^{1}$

Institutions

1 Department of Gastroenterology and Endoscopy

2 Division of Pathology, National Cancer Center Hospital East
\end{abstract}

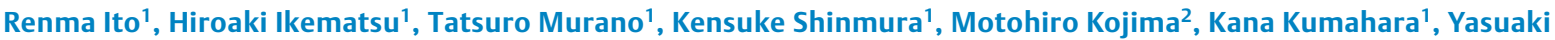
Furue $^{1}$, Hironori Sunakawa ${ }^{1}$, Tatsunori Minamide ${ }^{1}$, Daiki Sato ${ }^{1}$, Yoichi Yamamoto ${ }^{1}$, Kenji Takashima ${ }^{1}$, Yusuke Yoda ${ }^{1}$,

submitted 28.8.2020

accepted after revision $\quad 19.10 .2020$

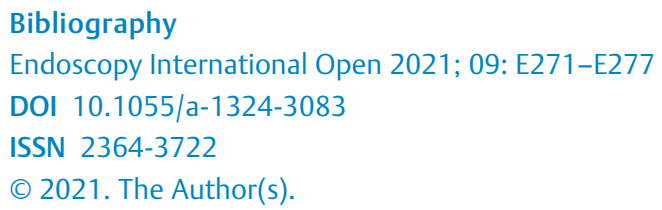
Commons Attribution-NonDerivative-NonCommercial License, permitting copying and reproduction so long as the original work is given appropriate credit. Contents may not be used for commecial purposes, or adapted, remixed, transformed or built upon. (https://creativecommons.org/licenses/by-nc-nd/4.0/)

Georg Thieme Verlag KG, Rüdigerstraße 14,

70469 Stuttgart, Germany

Corresponding author

Hiroaki Ikematsu, Department of Gastroenterology and Endoscopy, National Cancer Center Hospital East, 6-5-1, Kashiwanoha, Kashiwa, Chiba, 277-8577, Japan

Fax: +81-4-7131-4724

hikemats@east.ncc.go.jp

\section{ABSTRACT}

Background and study aims The Japan Narrow-band imaging (NBI) Expert Team (JNET) classification was proposed for evaluating colorectal lesions. However, it remains unknown whether the JNET classification can be applied to magnifying endoscopy with image-enhanced endoscopies other than NBI. This study aimed to compare the diagnostic ability of JNET classification by magnifying endoscopy with blue laser imaging (ME-BLI) and with ME-NBI.

Patients and methods We retrospectively assessed consecutive patients diagnosed per the JNET classification by ME-BLI (BLI group) or ME-NBI (NBI group) between March 2014 and June 2017. We compared the diagnostic value of JNET classification between the groups with one-to-one propensity score matching.

Results Four hundred and seventy-one propensity scorematched pairs of lesions were analyzed. In the BLI and NBI groups, the overall diagnostic accuracies were $92.1 \%$ and $91.7 \%$, respectively, and those for differentiating between neoplastic and non-neoplastic polyps were $96.6 \%$ and $96.8 \%$, respectively. The positive predictive value by each JNET classification in BLI vs. NBI group was $90.6 \%$ vs. $96.2 \%$ in Type 1, $94.3 \%$ vs. $94.6 \%$ in Type 2 A, $57.7 \%$ vs. $42.3 \%$ in Type $2 \mathrm{~B}$, and $100 \%$ vs. $91.7 \%$ in Type 3 . The negative predictive value was $97.0 \%$ vs. $96.9 \%$ in Type $1,88.1 \%$ vs. $82.8 \%$ in Type 2 A, $98.0 \%$ vs. $98.2 \%$ in Type 2B, and $98.5 \%$ vs. $98.7 \%$ in Type 3. No statistical difference in the diagnostic results was found between the groups.

Conclusions The diagnostic ability of the JNET classification by ME-BLI and ME-NBI was comparable, with the former also applicable for diagnosis of colorectal lesions.

\section{Introduction}

In recent decades, remarkable developments in endoscopic imaging such as magnifying endoscopy and image-enhanced endoscopies (IEEs) have brought about improvements in the qualitative and quantitative diagnoses of colorectal lesions [13].
Magnifying endoscopy with blue laser imaging (ME-BLI) and narrow-band imaging (ME-NBI) have been reported to be useful for differentiating neoplastic and non-neoplastic colorectal lesions and estimating the depth of colorectal carcinoma [4-7]. Recently, the Japan Narrow-band imaging Expert Team (JNET) classification was proposed and validated for the evaluation of colorectal lesions and currently provides unified diagnostic 
criteria for the prediction of pathological results by magnifying endoscopy with IEEs $[8,9]$. However, the JNET classification was originally developed using endoscopic findings by ME-NBI; thus, it is unclear whether the JNET classification is applicable to magnifying endoscopy with other IEEs.

BLI is another prevailing IEE, which uses a laser light source that can obtain bright and clear images [10]. ME-BLI has been reported to be useful for the optical diagnosis of colorectal lesions by recognizing the microvessels and surface structures of the lesions [6,7], and this diagnostic process is the same as the ME-NBI according to the JNET classification. Therefore, we hypothesized that the JNET classification by ME-BLI would give comparable diagnostic value for colorectal lesions to validated JNET classification by ME-NBI.

This retrospective study aimed to investigate the diagnostic ability of JNET classification by ME-BLI for colorectal lesions and compare it with ME-NBI.

\section{Patients and methods}

\section{Patients}

Clinical data from consecutive patients with colorectal lesions endoscopically diagnosed by ME-BLI or ME-NBI who underwent endoscopic or surgical resection at the National Cancer Center Hospital East from March 2014 to June 2017 were analyzed. The protocol was approved by the medical ethics committee of our hospital and written informed consent for diagnosis and treatment was obtained from all the patients before the procedures. The study was performed in accordance with the ethical principles based on the Declaration of Helsinki.

\section{Endoscopic system and settings}

We used high-resolution optical magnifying endoscopes (ECL590ZP, EC-L600ZP, EC-L600ZP7, Fujifilm Co, Tokyo, Japan; PCF-Q240ZI, PCF-Q260AZI, PCF-H290ZI, Olympus Medical Systems, Tokyo, Japan) and video processors (LASEREO, Fujifilm Co, Tokyo, Japan; EVIS LUCERA ELITE, Olympus Medical Systems, Tokyo, Japan). The BLI and NBI settings were fixed at surface structure enhancement level B- 6 and A-8 and at an adaptive index of color enhancement levels $C 2$ and $C 3$, respectively. The patients were inspected by either LASEREO or EVIS LUCERA ELITE system. The abbreviation of BLI has been used for blue laser imaging as well as blue light imaging, which was also developed by Fujifilm Co. and uses light-emitting diode (LED) light as the light source instead of LASER light [11]. In this study, we noted BLI as blue laser imaging by LASER light.

\section{Endoscopic procedure}

For bowel preparation, 1 to $2 \mathrm{~L}$ of polyethylene glycol solution was administered on the morning of the colonoscopy. Scopolamine butylbromide $(10 \mathrm{mg})$ or glucagon $(0.5 \mathrm{mg})$ was administered in the absence of contraindications, and midazolam $(0.03 \mathrm{mg} / \mathrm{kg})$ and $/$ or pethidine hydrochloride $(35 \mathrm{mg})$ were used for conscious sedation only when a patient complained of discomfort or pain. If a polyp was found, it was diagnosed by either ME-BLI or ME-NBI before resection.

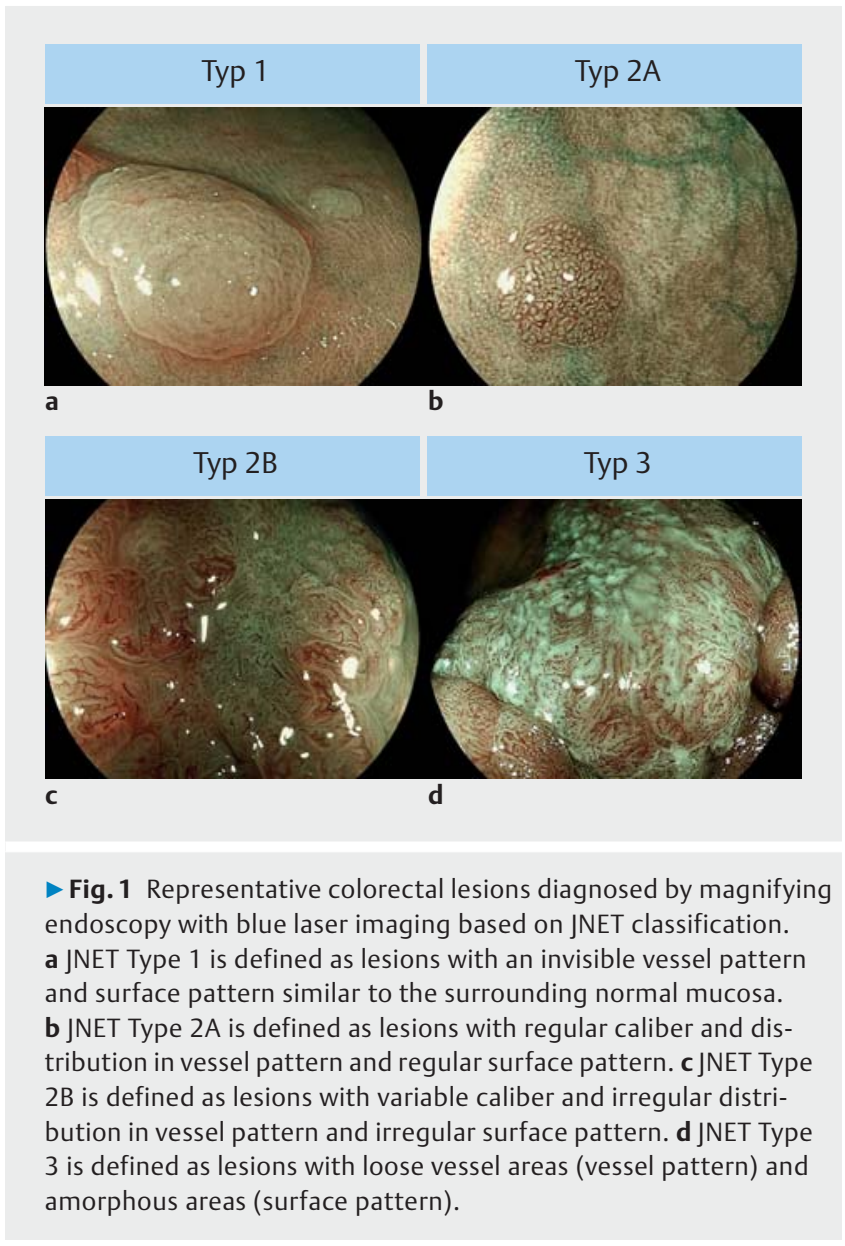

\section{Histopathological examination}

All resected specimens were retrieved and immediately fixed in $10 \%$ buffered formalin solution and examined histologically using hematoxylin and eosin staining. One clinical pathologist made the histological diagnosis according to the classification system of the World Health Organization [12]. In this study, deep submucosal invasive cancer (T1b) was defined as a tumor with a vertical submucosal invasion length $\geq 1000 \mu \mathrm{m}$, and shallow submucosal invasive cancer (T1a) was defined as a tumor with a vertical submucosal invasion length $<1000 \mu \mathrm{m}$.

\section{Evaluation of findings by ME-BLI and ME-NBI}

Endoscopists (ME-NBI diagnosis of $>500$ cases over 5 years) diagnosed the colorectal lesions with ME-NBI or ME-BLI and recorded their diagnoses before resection. Then, another experienced endoscopist blinded to the pathological results reevaluated all the lesions by assessing still images taken with ME-IEE. For lesions with ME-NBI, the diagnosis was given according to the JNET classification. Concerning lesions with ME-BLI, endoscopists adopted the concept of JNET classification and provided diagnosis by evaluating the microvessels and surface structures of the lesions. The typical correspondence between still images and JNET classification by ME-BLI is shown in > Fig. 1. When the diagnosis between the endoscopists who recorded the realtime diagnosis and another endoscopist who reevaluated still 
images differed, the final JNET diagnosis was decided after full consideration by another two experts.

\section{Study outcomes}

We analyzed the relationship between the JNET classification and the histopathological diagnosis and compared the diagnostic results between the $\mathrm{BLI}$ and the NBI groups in the propensity score-matched cohort. We judged the JNET diagnosis to be accurate when the lesions diagnosed as Type 1 were hyperplastic polyps (HP) or sessile serrated lesions (SSL), lesions diagnosed as Type 2A were low-grade dysplasia (LGD), lesions diagnosed as Type 2B were high-grade dysplasia (HGD) or shallow submucosal invasive cancer (T1a), and lesions diagnosed as Type 3 were deep submucosal invasive cancer (T1b). In this study, non-neoplastic polyps are defined as HP or SSL, and neoplastic polyps are defined as histology other than HP or SSL. We compared the following three items between each group: (1) the overall accuracy of the JNET classification; (2) the diagnostic accuracy of differentiating between neoplastic and non-neoplastic polyps; and (3) positive predictive value (PPV) and negative predictive value (NPV) for each type of JNET classification.

\section{Statistical analysis}

We performed a one-to-one matching analysis between the BLI group and the NBI group on the basis of estimated propensity scores of each lesion $[13,14]$. The propensity score approach addresses the selection bias that is inherent in retrospective observational studies such as the present study. Application of propensity score matching involved estimating the propensity score followed by matching of lesions in accordance with their estimated propensity score and comparison of outcomes in the matched lesions. To estimate the propensity score, we fitted a logistic regression model for the examination of ME-BLI as a function of clinicopathological factors of lesions including macroscopic type, lesion size, lesion location, and histological findings. Greedy matching (based on the estimated propensity score) was performed on the logit of the propensity score with a caliper width of 0.2 times the pooled standard deviation (SD) of the logit of the propensity scores for the cohort. Balance between the BLI group and the NBI group was assessed by calculating standard differences for which a difference of less than 0.10 was considered to indicate good balance. Nominal and ordinal variables were expressed as frequencies and percentages. Continuous variables (age and lesion size) are expressed as mean and SD. The $t$-test was used to compare the mean values of the two groups. Fisher's exact test or the Pearson $x^{2}$ test was used to analyze the categorical data and compare the proportions. All statistical tests were two-tailed, and significance was defined as $P<0.05$. We performed all statistical analyses with EZR (Saitama Medical Center, Jichi Medical University, Saitama, Japan), which is a graphical user interface for $R$ (The R Foundation for Statistical Computing, Vienna, Austria) [15].

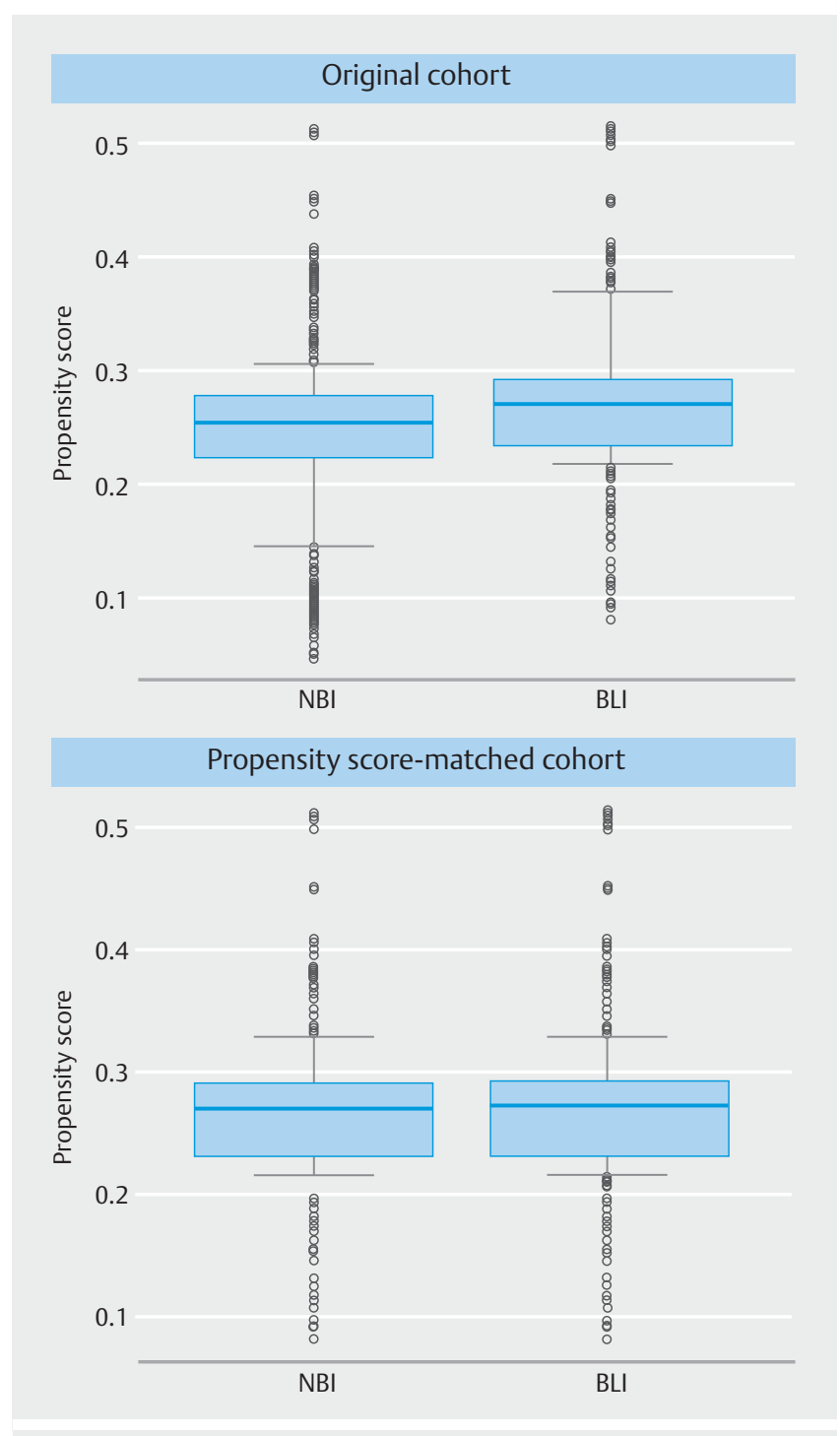

- Fig. 2 Distributions of propensity scores in the original (top) and propensity score-matched cohort (bottom).

\section{Results}

\section{Clinicopathological characteristics of colorectal lesions in the BLI and NBI groups}

We analyzed 246 patients with 499 lesions in the BLI group and 808 patients with 1459 lesions in the NBI group after excluding lesions that could not be evaluated due to poor image quality (37 lesions in the BLI group and 60 lesions in the NBI group). By one-to-one propensity score matching, 471 pairs of the BLI group and NBI group were selected. $>$ Fig. 2 shows the distributions of propensity scores in the unmatched and the matched group. The clinicopathological features of each group in the unmatched and matched cohort are shown in > Table 1. In the unmatched cohort, significant differences were found in the macroscopic type, the average size of lesions, and the proportion of histological findings between the BLI and the NBI groups. In the matched cohort, no significant differences were found be- 
- Table1 Clinicopathological features of each group in the original and propensity score-matched cohort.

\begin{tabular}{|c|c|c|c|c|c|c|c|c|}
\hline & \multicolumn{4}{|c|}{ Original cohort } & \multicolumn{4}{|c|}{ Propensity score-matched cohort } \\
\hline & BLI group & NBI group & $P$ value & SD & BLI group & NBI group & $P$ value & SD \\
\hline Lesions & 499 & 1459 & - & - & 471 & 471 & - & - \\
\hline \multicolumn{9}{|l|}{ Macroscopic type, number (\%) } \\
\hline - Polypoid & $321(64.5)$ & $843(57.8)$ & \multirow[t]{2}{*}{0.011} & \multirow[t]{2}{*}{0.049} & $299(63.5)$ & $293(62.2)$ & \multirow[t]{2}{*}{0.736} & \multirow[t]{2}{*}{0.01} \\
\hline - Non-polypoid & $178(35.5)$ & $616(42.2)$ & & & $172(36.5)$ & $178(37.8)$ & & \\
\hline - Lesion size $(\mathrm{mm}$, mean $\pm \mathrm{SD})$ & $7.9 \pm 9.02$ & $10.2 \pm 11.3$ & $<0.001$ & 0.23 & $7.97 \pm 9.14$ & $7.91 \pm 9.56$ & 0.92 & 0.006 \\
\hline \multicolumn{9}{|l|}{ Lesion location, number (\%) } \\
\hline - Right-sided colon & $279(55.9)$ & $844(57.8)$ & 0.482 & 0.014 & $277(58.8)$ & $272(57.7)$ & 0.792 & 0.008 \\
\hline - Left-sided colon & $163(32.7)$ & $472(32.4)$ & 0.941 & 0.002 & $154(32.7)$ & $157(33.3)$ & 0.89 & 0.005 \\
\hline - Rectum & $57(11.4)$ & $143(9.8)$ & 0.344 & 0.018 & $40(8.5)$ & $42(8.9)$ & 0.908 & 0.005 \\
\hline \multicolumn{9}{|l|}{ Histological findings, number (\%) } \\
\hline - HP/SSL & $65(13.0)$ & $103(7.1)$ & $<0.001$ & 0.86 & $42(8.9)$ & $39(8.3)$ & 0.816 & 0.008 \\
\hline - LGD & $394(78.9)$ & $1111(76.1)$ & 0.23 & 0.03 & $389(82.6)$ & $396(84.1)$ & 0.6 & 0.014 \\
\hline - HGD & $21(4.1)$ & $90(6.2)$ & 0.14 & 0.034 & $21(4.5)$ & $17(3.6)$ & 0.62 & 0.016 \\
\hline - T1a & $3(0.5)$ & 27 (1.9) & 0.079 & 0.046 & $3(0.6)$ & $2(0.4)$ & 1 & 0.01 \\
\hline . T1b & $16(3.6)$ & $128(8.8)$ & $<0.001$ & 0.077 & $16(3.4)$ & $17(3.6)$ & 1 & 0.004 \\
\hline
\end{tabular}

HP, hyperplastic polyp; SSL, sessile serrated lesion; LGD, low-grade dysplasia; HG, high-grade dysplasia.

tween the BLI group and the NBI group. In addition, all standardized differences in the matched cohort were less than 0.10 .

\section{Relationship between JNET classification and histology in the BLI group and the NBI group in the matched cohort}

The diagnostic agreement rates between the endoscopists who recorded the real-time diagnosis for colorectal lesions and the endoscopist who re-evaluated still images of the lesions were $96.8 \%$ in BLI group and $96.0 \%$ in NBI group. The relationship between the JNET classification by either ME-BLI or ME-NBI and histopathological diagnosis in the matched cohort are shown in $>$ Table 2. Histologically, majority of Type 1 lesions were identified as hyperplastic polyp/sessile serrated lesion (HP/SSL) in both groups (90.6\% in BLI group and $96.2 \%$ in NBI group). Similarly, majority of Type 2A lesions and Type 3 lesions were identified as LGD (94.3\% in BLI group and $94.6 \%$ in NBI group) and T1b (100\% in BLI group and $91.7 \%$ in NBI group), respectively. On the other hand, various histological types were included in Type 2B lesions in which LGD, HGD, T1a, and T1b were identified in $19.2 \%, 46.2 \%, 11.5 \%$, and $23.1 \%$ patients, respectively, in BLI group and 38.5\%, 34.6\%, 7.7\%, and $19.2 \%$ patients, respectively, in NBI group.

\section{Comparison of diagnostic ability between ME-BLI and ME-NBI in the matched cohort}

The diagnostic results of JNET classification between the BLI group and the NBI group in the matched cohort are shown in - Table 3 and $>$ Table 4 . The overall accuracy of the JNET clas- sification was comparable between the BLI and the NBI groups (92.1\% vs. 91.7\%, $P=0.905)$. Furthermore, the diagnostic accuracy of differentiating between neoplastic and non-neoplastic polyps was also comparable between the BLI group and the $\mathrm{NBI}$ group ( $96.6 \%$ vs. $96.8 \%, P=1.000)$. The PPV by each type of JNET classification in the BLI group vs. NBI group was $90.6 \%$ vs. $96.2 \%$ in Type $1,94.3 \%$ vs. $94.6 \%$ in Type $2 A, 57.7 \%$ vs. $42.3 \%$ in Type $2 \mathrm{~B}$, and $100 \%$ vs. $91.7 \%$ in Type 3 . The NPV in the BLI group vs. NBI group was $97.0 \%$ vs. $96.9 \%$ in Type 1 , $88.1 \%$ vs. $82.8 \%$ in Type 2 A, $98.0 \%$ vs. $98.2 \%$ in Type $2 B$, and $98.5 \%$ vs. $98.7 \%$ in Type 3 . No statistical differences were found in PPV and NPV between the BLI and the NBI groups.

\section{Discussion}

This retrospective study used a large cohort with a propensity score matching and provided the first evidence that the diagnostic value of ME-BLI based on the JNET classification is comparable to that of the ME-NBI in terms of the accuracy of the qualitative and quantitative diagnosis of the colorectal lesions. Our study showed that the overall diagnostic accuracy of the JNET classification was approximately $90 \%$ by both ME-BLI and $\mathrm{ME}-\mathrm{NBI}$, and notably, the diagnostic accuracy for differentiating between the neoplastic and non-neoplastic polyps was over $95 \%$ by both modalities. This result not only suggests the compatibility of ME-BLI and ME-NBI in the JNET classificationbased diagnosis but also emphasizes the high performance of the JNET classification for the diagnosis of colorectal lesions. 
- Table 2 Relationship between JNET classification and histological diagnosis of each group in the matched cohort.

\begin{tabular}{|c|c|c|c|c|c|c|}
\hline \multirow{2}{*}{$\begin{array}{l}\text { JNET } \\
\text { classification }\end{array}$} & \multirow[t]{2}{*}{ n (\%) } & \multicolumn{5}{|c|}{ Histological findings } \\
\hline & & HP/SSL & LGD & HGD & T1a & T1b \\
\hline \multicolumn{7}{|l|}{ BLI group } \\
\hline - Type 1 & $32(100)$ & $29(90.6)$ & $3(9.4)$ & - & - & - \\
\hline - Type 2A & $404(100)$ & $13(3.2)$ & $381(94.3)$ & $9(2.2)$ & - & $1(0.2)$ \\
\hline - Type 2B & $26(100)$ & - & $5(19.2)$ & $12(46.2)$ & $3(11.5)$ & $6(23.1)$ \\
\hline - Type 3 & $9(100)$ & - & - & - & - & $9(100)$ \\
\hline \multicolumn{7}{|l|}{ NBI group } \\
\hline - Type 1 & $26(100)$ & $25(96.2)$ & $1(3.8)$ & - & - & - \\
\hline - Type 2A & $407(100)$ & $14(3.4)$ & $385(94.6)$ & $7(1.7)$ & - & $1(0.2)$ \\
\hline - Type 2B & $26(100)$ & - & $10(38.5)$ & $9(34.6)$ & $2(7.7)$ & $5(19.2)$ \\
\hline - Type 3 & $12(100)$ & - & - & $1(8.3)$ & - & $11(91.7)$ \\
\hline
\end{tabular}

- Table 3 Comparison of diagnostic accuracy between the BLI and NBI groups in the matched cohort.

Accuracy, \% (95\% Cl)

\begin{tabular}{|l|l|l|l|}
\hline & BLI group & NBI group & P value \\
\hline Overall & $92.1(89.3-94.4)$ & $91.7(88.8-94.0)$ & 0.905 \\
\hline Neoplastic/non-neoplastic polyps & $96.6(94.5-98.0)$ & $96.8(94.8-98.2)$ & 1 \\
\hline BLI, blue laser imaging; NBI, narrow-band imaging. & & & \\
\hline
\end{tabular}

- Table 4 Comparison of PPV and NPV between the BLI and NBI groups in the matched cohort.

PPV, \% (95\% Cl)

\begin{tabular}{|l|c|c|c|}
\hline JNET & BLI group & NBI group & P value \\
\hline Type 1 & $90.6(74.9-98.0)$ & $96.2(80.3-99.9)$ & 0.620 \\
\hline Type 2A & $94.3(91.5-96.3)$ & $94.6(91.9-96.5)$ & 0.879 \\
\hline Type 2B & $57.7(36.9-76.6)$ & $42.3(23.3-63.0)$ & 0.406 \\
\hline Type 3 & $100(96.3-100)$ & $91.7(61.5-99.7)$ & 1 \\
\hline PPV, \% (95\% Cl) & & & P value \\
\hline JNET & BLI group & NBI group & 1 \\
\hline Type 1 & $97.0(94.8-98.4)$ & $96.9(94.7-98.2)$ & 0.461 \\
\hline Type 2A & 88.1 (77.8-94.7) & $82.8(71.3-91.0)$ & 1 \\
\hline Type 2B & $98.0(96.1-99.0)$ & $98.2(96.4-99.2)$ & 1 \\
\hline Type 3 & $98.5(96.9-99.3)$ & $98.7(97.1-99.5)$ & \\
\hline JNET,Japan Narrow-band Imaging Expert Team; BLI, blue laser imaging; NBI, narrow-band imaging.
\end{tabular}

To date, several IEEs such as NBI, i-Scan (Pentax Co., Tokyo, Japan), and BLI have been developed for the evaluation of colorectal lesions [16]. BLI is a unique IEE in that its system uses semiconductor laser as the light source instead of a xenon lamp as used by other systems. Although the system is different, BLI is designed to visualize the microvessels and surface structures with high contrast by arranging the ratio of blue light laser (wave length $410 \pm 10 \mathrm{~nm}$ ) over white light laser (wave 
length $450 \pm 10 \mathrm{~nm}$ ) for narrow-band observation [10], which has the same concept for image enhancement as NBI which allows the narrow-band observation using the optical filters. Several papers have shown that BLI or NBI combined with magnifying endoscopy is useful for the qualitative and quantitative diagnosis of the colorectal lesions [4-7], but no report has compared the diagnostic ability of ME-BLI and ME-NBI using the same diagnostic criteria, presumably because of the lack of a unified diagnostic system before the validation of the JNET classification, and the difficulty in gathering a large cohort with high-quality images by both ME-BLI and ME-NBI which enables the endoscopists to conduct the retrospective evaluations.

The major strength of this study was that we successfully obtained a large cohort and used propensity score matching in consecutive patients who were inspected by either the BLI group or the NBI group. Furthermore, they had high-quality magnified images for the retrospective assessment, which is feasible only in a high-volume center with endoscopists who have been well trained in magnifying endoscopy. This study offers two major points of clinical importance: first, the diagnostic results of the JNET classification by two modalities, ME-BLI and $\mathrm{ME}-\mathrm{NBI}$, were comparable judging by the almost equal PPV and NPV of all JNET types. This result will expand the versatility of the JNET classification to ME-BLI in the countries where the LASEREO system is as prevalent as the EVIS LUCERA ELITE system like in Japan, and the JNET classification may be applied to other IEEs such as i-Scan and the recently developed blue light imaging, which uses four LEDs as the light source and has been released by Fujifilm Co. in some areas, including the United States and Europe, where the LASEREO system has not been approved for use [17]. Second, we confirmed the reproducibility of the diagnostic performance of the JNET classification. Notably, over $90 \%$ of the Type 1 cases were accurately diagnosed as neoplastic or non-neoplastic lesions. The NPV of Type 1 in both $\mathrm{BLI}$ and $\mathrm{NBI}$ groups was within the standard set by the American Society for Gastrointestinal Endoscopy for NPV (90\% or more) for differentiating neoplastic and non-neoplastic colorectal lesions [16]. Furthermore, more than $90 \%$ of the lesions diagnosed as Type 2A were LGD, and almost all lesions diagnosed as Type 3 were T1b in both ME-BLI and ME-NBI. Conversely, lesions diagnosed as Type $2 \mathrm{~B}$ contained various histologies from LGD to T1b, reflecting the low diagnostic value for Type $2 \mathrm{~B}$. These results were completely consistent with previous reports by Sumimoto et al. $[18,19]$ and Komeda et al. [20], which highlight the idea that pit pattern diagnosis should be performed for lesions of Type 2B to predict the histology more precisely.

This study has some limitations. First, it was retrospective and single-center. A prospective non-inferiority trial is necessary to show the equivalency of diagnostic ability by ME-BLI to one by ME-NBI. Second, we used still images for retrospective JNET diagnosis, which is a different diagnostic process from that employed in clinical practice. Third, there could be selection bias for colorectal lesions between the BLI group and the NBI group, although consecutive patients were assigned to each group with no intervention by the investigators. Indeed, we showed that the BLI group had a lower proportion of non- polypoid lesions and a smaller average size of lesions than the $\mathrm{NBI}$ group. Therefore, we used propensity score matching in this study to reduce selection bias. However, there may still be some bias from unobserved differences. Fourth, we did not assess the diagnostic ability of ME-BLI and ME-NBI using the same target lesions. For a direct comparison, it would be ideal to examine diagnosis for the same lesion by the same endoscopist, but this type of study design is quite difficult in a real clinical setting. Thus, we compensated for this shortfall by including a large cohort, a unified setting of ME-BLI and ME-NBI in taking the images, and objectivity in evaluation of the JNET diagnosis.

\section{Conclusion}

In conclusion, the diagnostic ability of JNET classification with ME-BLI was comparable with that of ME-NBI. Although a prospective study is warranted to perform an unbiased analysis, this retrospective study in a propensity score-matched cohort suggests that the JNET classification, originally developed with ME-NBI, could also be applied to diagnosis of colorectal lesions using ME-BLI, which broadens the utility of the JNET classification, leading more endoscopists to make a more accurate diagnosis and provide more appropriate subsequent treatment.

\section{Competing interests}

The authors declare that they have no conflict of interest.

\section{References}

[1] Machida $\mathrm{H}$, Sano $\mathrm{Y}$, Hamamoto $\mathrm{Y}$ et al. Narrow band imaging for differential diagnosis of colorectal mucosal lesions: a pilot study. Endoscopy 2004; 36: 1094-1098

[2] Sano Y, Horimatsu T, Fu Kl et al. Magnifying observation of microvascular architecture of colorectal lesions using a narrow-band imaging system. Dig Endosc 2006; 18: S44-S51

[3] Tanaka S, Hirata M, Oka S et al. Clinical significance of narrow band imaging (NBI) in diagnosis and treatment of colorectal tumor. Gastroenterol Endosc 2008; 50: 1289-1297

[4] Sano Y, Ikematsu H, Fu KI et al. Meshed capillary vessels by use of narrow-band imaging for differential diagnosis of small colorectal polyps. Gastrointest Endosc 2009; 69: 278-283

[5] Ikematsu H, Matsuda T, Emura F et al. Efficacy of capillary pattern type IIIA/IIIB by magnifying narrow band imaging for estimating depth of invasion of early colorectal neoplasms. BMC Gastroenterol 2010; 10: 33

[6] Yoshida N, Yagi N, Inada Y et al. Ability of a novel blue laser imaging system for the diagnosis of colorectal polyps. Dig Endosc 2014; 26: 250-258

[7] Yoshida N, Hisabe T, Inada Y et al. The ability of a novel blue laser imaging system for the diagnosis of invasion depth of colorectal neoplasms. J Gastroenterol 2014; 49: 73-80

[8] Sano Y, Tanaka S, Kudo SE et al. Narrow-band imaging (NBI) magnifying endoscopic classification of colorectal tumors proposed by the Japan NBI Expert Team. Dig Endosc 2016; 28: 526-533

[9] Iwatate M, Sano Y, Tanaka S et al. Validation study for development of the Japan NBI Expert Team classification of colorectal lesions. Dig Endosc 2018; 30: 642-651 
[10] Kaneko K, Oono Y, Yano T et al. Effect of novel bright image enhanced endoscopy using blue laser imaging (BLI). Endosc Int Open 2014; 2: E212-E219

[11] Yoshida N, Dohi O, Inoue K et al. Blue laser imaging, blue light imaging, and linked color imaging for the detection and characterization of colorectal tumors. Gut Liver 2019; 13: 140-148

[12] Bosman FT, Carneiro F, Hruban RH et al. WHO Classification of tumors of the digestive system. 4th ed. IARC; 2010

[13] Rosenbaum PR, Rubin DB. Constructing a control group using multivariate matched sampling methods that incorporate the propensity score. Am Stat 1985; 39: 33-38

[14] Landrum MB, Ayanian JZ. Causal effect of ambulatory specialty care on mortality following myocardial infarction: a comparison of propensity score and instrumental variable analyses. Health Serv Outcome Res Meth 2001; 2: 221-245

[15] Kanda Y. Investigation of the freely available easy-to-use software 'EZR' for medical statistics. Bone Marrow Transplant 2013; 48: 452458

[16] Abu Dayyeh BK, Thosani N et al. ASGE Technology Committee. ASGE Technology Committee systematic review and meta-analysis asses- sing the ASGE PIVI thresholds for adopting real-time endoscopic assessment of the histology of diminutive colorectal polyps. Gastrointest Endosc 2015; 81: 502.e1-16

[17] Bisschops R, Hassan C, Bhandari P et al. BASIC (BLI Adenoma Serrated International Classification) classification for colorectal polyp characterization with blue light imaging. Endoscopy 2018; 50: 211-222

[18] Sumimoto K, Tanaka S, Shigita K et al. Clinical impact and characteristics of the narrow-band imaging magnifying endoscopic classification of colorectal tumors proposed by the Japan NBI Expert Team. Gastrointest Endosc 2017; 85: 816-821

[19] Sumimoto K, Tanaka S, Shigita K et al. Diagnostic performance of Japan NBI Expert Team classification for differentiation among noninvasive, superficially invasive, and deeply invasive colorectal neoplasia. Gastrointest Endosc 2017; 86: 700-709

[20] Komeda Y, Kashida H, Sakurai T et al. Magnifying narrow band imaging (NBI) for the diagnosis of localized colorectal lesions using the Japan NBI Expert Team (JNET) classification. Oncology 2017; 93: 4954 